\title{
Ethics in Psychiatric Research: Issues and Recommendations
}

\author{
Altaf Ahmad Malla ${ }^{1}$, Nasir Mohammad Bhat ${ }^{2 *}$
}

\section{ABSTRACT}

Psychiatric research has increased remarkably over recent decades to help in understanding the current trends and better therapeutic options for illness. On the other hand, there is also a trend toward higher rates of retraction of published papers in the recent years. Ethics is required to maintain and increase the overall quality and morality of research. Psychiatric research faces several unique ethical challenges. Ethical guidelines are very important tool of research which safeguards participants; however, there is a dearth of such guidelines in India. The present paper aims to review available ethical issues and guidelines pertaining to psychiatric research. A search was conducted on Pubmed using search terms (e.g., "ethics," “psychiatry," "research”). Relevant studies were selected for the review after manual screening of title/abstract. Additional sources were referred to using cross references and Google Scholar. Psychiatric research has several important ethical issues which are different from other medical disciplines. These issues are related to informed consent, confidentiality, conflict of interest, therapeutic misconception, placebo related, vulnerability, exploitation, operational challenges, among others. The current paper has made several recommendations to deal with ethical challenges commonly faced in psychiatric research. The ethical guidelines are utmost needed for Indian psychiatric research. Specific guidelines are lacking pertaining to psychiatric research. The issues and recommendations merit a further discussion and consideration.

Keywords: Ethics, mental health, psychiatry, research

The term "ethics” has been derived from the ancient Greek words "ethos," which implies to understand right and wrong. ${ }^{[1]}$ It refers moral standards and values that a person carries within himself. ${ }^{[2]}$ Research is defined as "any systematic investigation designed to develop or contribute to generalizable knowledge."[2]

The World Health Organization (WHO) estimates that $10 \%$ of world population is suffering from some psychiatric illness and $25 \%$ of person experience some psychiatric illness during their

\footnotetext{
${ }^{1}$ Institute of Mental Health and Neurosciences, Kashmir, India

${ }^{2}$ Institute of Mental Health and Neurosciences, Kashmir, India

*Responding Author

(C) 2016, A Malla, N Bhat; licensee IJIP. This is an Open Access Research distributed under the terms of the Creative Commons Attribution License (http://creativecommons.org/licenses/by/2.0), which permits unrestricted use, distribution, and reproduction in any Medium, provided the original work is properly cited.
} 


\section{Ethics in Psychiatric Research: Issues and Recommendations}

lifetime. ${ }^{[3,4]}$ According to a review by Math and Srinivasaraju, at least $20 \%$ of population in India is suffering from some psychiatric illness. ${ }^{[5]}$ Five out of ten leading cause of disability are due to mental illness, for example, depression, alcohol abuse, bipolar mood disorder, schizophrenia, and obsessive-compulsive disorder (OCD). ${ }^{[6]}$ The continued suffering and disability due to mental illness calls for newer treatments and continued research into field of psychiatry.

Psychiatric disorders itself carry significant stigma, myths, and biases. Causation of psychiatric disorders has varied beliefs as described by cultural psychiatrist as explanatory models of illness that includes moral model, religious model, magical or supernatural model, medical model, and psychosocial stress model. ${ }^{[7]}$

Considering various issues involved in psychiatric disorders and research, ethics plays crucial role in protecting rights of persons with mental illness and simultaneously safeguarding the interest of researchers. Ethical guidelines in research help maintain transparency and accountability during research. Therefore, various ethical guidelines have been put forward by national and international organizations for different groups of researchers.

Historically, ethical abuse in human research was noticed in experiments conducted by Nazi doctors on convicts, following which the first international standard for ethical conduct of research was framed, the Nuremberg Code in $1947 .{ }^{[8,9]}$ It was based on ten principles focusing on voluntary consent, right to withdraw from the study, and prohibited studies causing harm to patients. The World Medical Association also adopted a set of guidelines known as the Declaration of Helsinki which consisted 32 principles, which focused on informed consent, confidentiality, vulnerable population, and requirement of a research protocol stating scientific rationale of the study which need to be reviewed by the Ethics Committee ${ }^{[10]}$ Later on, following infamous Syphilis study at Tuskegee in 1972, the National Commission for the Protection of Human Subjects of Biomedical and Behavioral Research was founded in 1974. ${ }^{[11]}$ In 1979, the commission drafted the Belmont Report - Ethical Principles and Guidelines for the protection of human subjects of research. Due to increase in research conducted by pharmaceutical companies in underdeveloped and developing countries, "International Ethical Guidelines for Biomedical Research Involving Human Subjects" were developed in 1982 by the Council for International Organizations

In context of ethics in clinical psychiatry, in 1970, the American Psychiatric Association first time appointed committee to develop a code of ethics. Later in 1977, the World Psychiatric Association developed code of ethics for clinical practice known as "Declaration of Hawaii," which was later adopted by a committee in 1989 at Annual National Conference of Indian Psychiatric Society at Cuttack, Orissa (India). The code was based on principles of responsibility, competence, benevolence, moral standard, patient welfare, and confidentiality. ${ }^{[15]}$ However, no guidelines are available in context of psychiatry research. Further, India, which is 


\section{Ethics in Psychiatric Research: Issues and Recommendations}

the second largest populated country and houses one-seventh of the worlds' total population, is rapidly becoming a research hub for human research. Following globalization and industrialization, multinationals have an emerging interest for research in this area. There has been growing concern that research in developing countries, such as India, with lack of sufficient ethical guidelines will further lead to exploitation and injustice. ${ }^{[16]}$ It has been noticed that journals from low and middle income countries (LAMIC) often publish poor quality of research compared to international journals. ${ }^{[17]}$ Balhara and Mishra (2015) reviewed retraction rates of articles published on psychiatric illness and reported that the retraction rate was gradually increasing from 3.56 per 10,000 published articles on mental disorders in 2005 to 49.25 per 10,000 research, accountability and transparency - persons involved in research must share their contribution and conflict of interest, availability - the findings of research should be published so that it could add to the available literature. Balhara and Mishra (2015) reviewed retraction rates of articles published on psychiatric illness and reported that the retraction rate was gradually increasing from 3.56 per 10,000 published articles on mental disorders in 2005 to 49.25 per 10,000 of Medical Sciences in association with WHO. ${ }^{[12]}$ Strech et al. reported among 123 psychiatry journals mentioning International Committee of Medical Journal Editors (ICMJE) and COPE guidelines, about half of the journals' editorial policy required ethical review (54\%) and informed consent (58\%) whereas $14 \%$ and $19 \%$ demanded the reporting of these issues in the manuscript, respectively. ${ }^{[13]}$

\section{CURRENT SCENARIO}

In India, we have been left with few regulations to protect the human subjects from exploitation, which are either too broadly or too narrowly applied/implemented without serving any purpose. All clinical trials in India require prior permission from the Drug Controller General, India, and approval by the concerned hospital's Ethics Committees. The Ethical Committee would be a team of 5-12 persons, comprising doctors from the institution, independent observers, ethicists, and lawyers. ${ }^{[14]}$ Indian Council for Medical Research has published a detailed set of guidelines in 2000 titled "Ethical Guidelines for Biomedical Research" on human subjects, which seeks to update the policy statement on "Ethical Considerations Involved in Research on Human Subjects" that was brought out by the Indian Council of Medical Research (ICMR). ${ }^{[14]}$

In context of ethics in clinical psychiatry, in 1970, the American Psychiatric Association first time appointed committee to develop a code of ethics. Later in 1977, the World Psychiatric Association developed code of ethics for clinical practice known as "Declaration of Hawaii," which was later adopted by a committee in 1989 at Annual National Conference of Indian Psychiatric Society at Cuttack, Orissa (India). The code was based on principles of responsibility, competence, benevolence, moral standard, patient welfare, and confidentiality. ${ }^{[15]}$ However, no guidelines are available in context of psychiatry research. Further, India, which is the second largest populated country and houses one-seventh of the worlds' total population, is rapidly becoming a research hub for human research. Following globalization and 


\section{Ethics in Psychiatric Research: Issues and Recommendations}

industrialization, multinationals have an emerging interest for research in this area. There has been growing concern that research in developing countries, such as India, with lack of sufficient ethical guidelines will further lead to exploitation and injustice. ${ }^{[16]}$ It has been noticed that journals from low and middle income countries (LAMIC) often publish poor quality of research compared to international journals. ${ }^{[17]}$ Balhara and Mishra (2015) reviewed retraction rates of articles published on psychiatric illness and reported that the retraction rate was gradually increasing from 3.56 per 10,000 published articles on mental disorders in 2005 to 49.25 per 10,000 published articles on mental disorders in 2012 and that the retraction rates were higher in articles from LAMIC and predominantly from Asian countries compared to non-Asian countries. ${ }^{[18]}$ Analysis of randomized controlled trials published in 65 Indian medical journals in 2004-2005 reported that only one-third of journals adopted CONSORT guidelines and among those which adopted quality of reporting for reducing bias was suboptimal. ${ }^{[19]}$ Chaturvedi and Somashekar reviewed ethical aspects of articles published in Indian Journal of Psychiatry and reported that informed consent was mentioned in 51\% studies in 2000 and 82\% in 2007, whereas ethical approval was mentioned in only $2 \%$ studies in 2000 and $25 \%$ studies in 2007 . This raises doubts on ethical standards of research in Indian context. ${ }^{[20]}$ However, there is also concern that by imposing unnecessary and expensive regulatory burdens, scientific research may be hampered. Therefore, people with mental illness are vulnerable, but it does not imply to preclude them from human research. Neglecting people with mental illness itself raises concern for injustice and discrimination. ${ }^{[21]}$ Therefore, a balanced guidelines for psychiatry research is required. Due to above-mentioned rationale, this paper discusses ethical issues in psychiatry in research.

\section{CARDINAL PRINCIPLES OF ETHICS IN BIOMEDICAL RESEARCH}

The four cardinal principles of biomedical ethics are autonomy, nonmaleficence, beneficence, and justice. ${ }^{[22]}$ Respect for autonomy states that acting intentionally after providing sufficient information and time to decide. To ensure autonomy of participants, they must understand the potential risks and benefits of participation and need to be free from any undue influence/coercion which might affect their decision to participate. Participants should be treated as autonomous agents and persons with diminished autonomy (i.e., person with mental illness, prisoners and children) are entitled to additional protection. ${ }^{[15]}$ Nonmaleficence implies minimization of harm which can be achieved by careful decision-making and having adequate training. Beneficence deals with maximization of benefits to promote well-being of participant and society. Justice ensures equitable distribution of social benefits so that burdens and benefits of research are shared fairly and equitably by society. ${ }^{[23,24]}$

Apart from these cardinal principles, other principles are as follows: essentiality - stating rationale for the research based on available scientific knowledge, confidentiality - based on principle of nonmaleficence, competence - researcher needs to be competent in area of research 


\section{Ethics in Psychiatric Research: Issues and Recommendations}

as well as ethical considerations in particular research, accountability and transparency - persons involved in research must share their contribution and conflict of interest, availability - the findings of research should be published so that it could add to the available literature.

\section{ETHICAL ISSUES IN PSYCHIATRIC RESEARCH}

\section{Diagnosis}

The diagnosis of mental illness is based on diagnostic criteria consisting of subjective symptoms and behavior observation, which is constantly being revised from time to time to improve diagnostic classification. Demarcation between normal and abnormal behavior often becomes

hazy. ${ }^{[25]}$ Symptoms used in diagnosis can also be seen normally, but there is only a difference in frequency or severity. With advent of research in psychiatry, the number of diagnosis is on rise in newer classificatory systems example 106 in Diagnostic and Statistical Manual of Mental Disorders, First Edition (DSM I) (1952), 182 in DSM II (1968), 265 in DSM III (1980), 297 in DSM IV (1994), approximately 340 in DSM V (2013). In addition, newer classificatory system have either recategorized or new diagnosis (example as found in DSM V OCD, disruptive mood dysregulation disorder, somatic symptom disorder, illness anxiety disorder, hoarding disorder, excoriation [skin-picking disorder], disinhibited social engagement disorder, avoidant/restrictive food intake disorder, social [pragmatic] communication disorder, restless leg syndrome, binge eating disorder, premenstrual dysphoric mood disorder, mild neurocognitive disorder, caffeine withdrawal, cannabis withdrawal, factitious disorder by proxy, rapid eye movement sleep behavior disorder, etc.). Such recategorization is rarely seen other branches of medical fraternity. The diagnosis of mental illness to-date is largely based on symptomatology and observations, and there relatively lack of investigations and laboratory markers to precisely diagnose mental illness. Due to which mental conditions are sometimes wrongly diagnosed or differently diagnosed by different clinicians and researchers. Labeling a person with mental illness poses person psychological and social consequences, such as shame, blame, secrecy, exclusion, danger, discrimination, and stigma, leading to isolation and rejection of that person in all aspects of their lives. $^{[26]}$

\section{Recommendations}

Diagnosing a person with mental illness while recruitment in research, one must keep in mind about various psychological and social consequences of diagnosis over that person. If any matter of doubt in diagnosis, the person's interest must be favored over research interest.

\section{Researcher participant relationship}

Mental illness itself affects persons' cognitive functions, emotions, and behavior leading to issues of decision-making capacity, transference, and countertransference. Usually researchers are highly respected and given authority by affected persons and caregivers. This may consequently lead exploitation of rights of persons with mental illness. Due to such relationships, researchers may take advantage over issues of informed consent, providing wrong information, 


\section{Ethics in Psychiatric Research: Issues and Recommendations}

therapeutic misconception, unnecessary and prolonged investigations, exposure to harm related to investigations, pharmacological and nonpharmacological treatment, and malicious intimate relationship.

\section{Recommendations}

The relationship between the investigator and participants should be based on honesty, trust, and respect. ${ }^{[23]}$ Hence, any research group who wish to conduct any research on persons with mental illness must consist of at least one researcher who is competent and trained to understand rights of person with mental illness. Therefore, appropriate measures should be taken to provide brief training in ethics to researchers by Psychiatry Department of medical colleges and mental health institution.

\section{Involuntary treatment}

Mental illness often compromises persons' decision-making capacity, insight related to need for treatment. The treatment aims at modifying behavior, perceived as an implied threat because the psychiatric treatment occasionally may be utilized for controlling behavior for certain vested interests. This necessitates physicians and psychiatrists to execute principals of beneficence and nonmaleficence to treat patients making balance with autonomy of patients. Recruiting such patients for research raises issues of informed consent, decision-making capacity, autonomy. In addition, pharmacological trial of doubtful or no proven efficacy to such patients also raises issues of beneficence and nonmaleficence.

\section{Recommendations}

In such situations, researchers should respect advance directives and consent procedure as recommended below under issues of informed consent.

\section{Electroconvulsive therapy}

Research related to electroconvulsive therapy (ECT) faces issues of informed consent as the patients planned for ECT usually lack decision-making capacity. Unmodified ECT inflicts pain and cause discomfort to the patients. Further, indication of ECT is either based on severity of illness or treatment resistance; therefore, issues of beneficence and nonmaleficence arise on conducting sham procedures on severely ill or resistant cases.

\section{Recommendations}

Informed consent needs to be taken from legal guardian keeping into best interest of rights of patient. Research related to unmodified ECT should never be performed assuming that no person with full decision-making capacity would ever consent for any painful procedure without anesthesia. Sham procedures on severely ill or resistant cases should never be performed. 
Ethics in Psychiatric Research: Issues and Recommendations

\section{Confidentiality}

Confidentiality refers to researcher's responsibility of not disclosing information learned during research to anyone without the patient's permission. Many times, some new neurobiological and genetic markers of mental illness are investigated with poorly established sensitivity and specificity. On one hand, nondisclosure of results of person's investigations prejudices persons' right, whereas disclosure of results of unproven markers also bears ethical issues. Further, Right to Information (RTI) Act 2005 of India has raised an issue of confidentiality. Apart from this, several issues of confidentiality are seen in context of mandatory legal obligations in POCSO Act 2012 (i.e., if researcher gets knowledge child sexual abuse in his participant), tarasoff duty (i.e. if researcher gets knowledge of patient likely to harm other), disclosure of HIV status to spouse or fiancée.

\section{Recommendations}

Always researcher must establish trust with participants by explaining about confidentiality of information gathered during interview. Consent for legal obligations regarding privilege communications needs to be obtained beforehand. Privacy should always be maintained while gathering information from the participants, interview in front of others including relatives should be avoided. The participant's records should not lie unguarded where they could be accessed by persons outside of the research team. Further, unique identification code should be generated for each participant, which shall be used in case records/ pro forma rather than using name, address, and other identification details. Any publication should never contain identification details of participants. During disclosure of results of any investigation to the participants, relative strength and weakness of the investigation need to be emphasized. If any result that on disclosure bears physical, psychological, or social consequences, then same need to be referred to a clinician with explicit permission of consentee for proper assessment and disclosure of facts by a clinician. Confidential information if requested by family or employers under RTI Act, 2005 should be provided only after explicit permission is obtained from the participant or consentee. Wherever mandatory legal obligations are to be fulfilled, participants or consentee need to be informed about the same and information disclosed should contain only the fact regarding the issue, based on principals of nonmaleficence, must always be bonafide and only to the concerned authority. Psychosocial support needs to be provided to the participant wherever required. If harm to others is suspected, same need to be informed to consentee and participants need to be referred to clinician for assessment and management.

\section{Informed consent}

People with mental illness face more issues in consent process compared to other medical illness and healthy person. ${ }^{[27]}$ The concept of informed consent is based on three aspects:

a. Providing information - Consist details of information about the research protocol. Research subjects often fail to appreciate distinction between the imperatives of clinical research and of ordinary treatment. ${ }^{[28]}$ Most of the times, participants have misconception 


\section{Ethics in Psychiatric Research: Issues and Recommendations}

about degree to which their treatment will be individualized to meet their specific needs, likelihood of benefit from participation, goals of the researchers in conducting the projects. ${ }^{[2]}$ Appelbaum, 2004, reviewed 44 clinical trials and reported therapeutic misconception in $62 \%$ studies. High therapeutic misconception has been reported in genetic studies $(74 \%)^{[30]}$ and psychiatric research $(69 \%)^{[31]}$

b. Competence - Ability of participants to understand the information and make rational decisions. Even though information provided to the participants is adequate, consent in psychiatry research is complicated by inability of persons suffering from mental illness to process the provided information adequately. This may be either due to lack of awareness of illness or due to cognitive changes that occur during illness. Several tools are available to assess decision-making capacity as MacArthur Treatment Competence Assessment Tool for Clinical Research (MacCAT-CR) ${ }^{[32]}$ and University of California Brief Assessment of Capacity to Consent ${ }^{[33]}$

c. Autonomy - The concept of autonomy in research is established when the patient can take its autonomous decisions without being influenced by the disease process, cultural factors, or other extraneous factors. It consists of two main aspects: (1) choice of participants and (2) noncoercion/noninducement. ${ }^{[34]}$ Autonomy is the main decision-making factor for participation in any research. Concept of autonomy varies in different cultural settings.

Only $20 \%-30 \%$ of patients lack decision-making capacity, ${ }^{[35]}$ further it is not a static phenomenon rather a dynamic as its status can change from time to time. This decisional impairment depends on diagnosis, severity of illness, and response to treatment. ${ }^{[23]}$

\section{Recommendations}

At the start of the study, an information leaflet explaining the research project in lay terms should be provided to each participant. The information booklet should contain information about the research including title, rationale, aims, research design and methodology, various investigation, and procedure participant likely to undergo during research, associated harms and compensation in research, estimated duration likely to be spent in research, any incentives provided for participation. All information need to be explained in language that is understandable by the participants.

Consent forms are also required to be printed in the language that is understandable by the participants. Two copies of the consent forms must be signed by the participant and the researcher, and one copy needs to be given to the participant.

In situations where competency is initially compromised, advance directives if available need to be respected,. In case of non availability of advance directives, consent needs to be taken from 


\section{Ethics in Psychiatric Research: Issues and Recommendations}

the legally authorized representative or caregiver taking into account, best interest of the patient. MacCAT-CR can be used to measure capacity to consent research. ${ }^{\text {[32] }}$

The consent process should be continuous and not one time. Decision-making capacity may improve by providing repeated information using different methods as conversation, lectures, pamphlets, articles, group discussion, videos, etc. ${ }^{[36,37]}$ Whenever the patient regains competence, advance directive needs to be taken and consent to remain in the research needs to be taken. ${ }^{[23]}$ If such representative is not available and recruitment cannot be delayed, then the study may proceed without informed consent, provided specific reason for inability to consent have been stated in protocol, and approved by the Ethics Committee. Consent to remain in the study should be taken as soon as possible. If a participant is incompetent to consent but able to assent the decision, then assent must be taken in addition to consent of legal representative. Any dissent should be respected. ${ }^{[38]}$

Participants could withdraw from the study at any point during the study or within a 2-week period after the completion of their participation in the study. ${ }^{[39]}$

\section{Conflict of interest}

Conflict of interest is a set of conditions in which professional judgment concerning a primary interest (such as patients' welfare or validity of research) tends to be unduly influenced by a secondary interest (such as financial gain or professional gain).

Similar to other specialties of medical science, conflict of interest has also been noticed in psychiatric research. These may occur at various level as pharmaceutical companies for the development of new drugs and marketing whereas investigators for direct and indirect financial gains by association with sponsors or for academic interest. ${ }^{[40]}$ Fang and Casadevall (2011) have reviewed 2047 retracted research articles indexed by PubMed and reported 67.4\% retraction due to misconduct (fraud [43.4\%], duplicate publication [14.2\%], and plagiarism [9.8\%]). Retraction for fraud was reported to increase by ten times since 1975. Plagiarism and duplicate publication were reported maximum from China and India. Retraction due to fraud and error was found more among journals with higher impact factor, whereas plagiarism and duplicate publications were more among journal with lower impact factor. ${ }^{[41]}$ In addition, journals, publishers, and conferences have now grown rampantly under the influence of pharmaceutical companies ${ }^{[42]}$ and unprofessionally publishing the research for their own financial gains. ${ }^{[43]}$ Often young researchers become prey of such journals so as to boost their academic career. Due to lack resources for high-quality research in developing and underdeveloped countries, they often become prey of these predatory journals. 


\section{Ethics in Psychiatric Research: Issues and Recommendations}

\section{Recommendations}

The Ethical Committee of each institute should be framed after formal approval from national regulatory bodies, for example, ICMR. The Ethical Committee is not only responsible for approving the initiation of studies but also for regularly monitoring, the approved researches are compliance with the ethical guidelines. Concerned Ethical Committee should equally be held responsible for any breach in ethical standards during the study period. Following guidelines may be adopted for submitting the protocol such as CONSORT guidelines for Randomized Clinical Trials, ${ }^{[44,45]}$ STROBE for observational studies, such as cross-sectional studies, casecontrol studies, and cohort studies; ${ }^{[46,47]}$ STARD guidelines for studies evaluating diagnostic accuracy as sensitivity and specificity; ${ }^{[48]}$ COREQ guidelines ${ }^{[49]}$ for quality reporting of interviews and focused group discussion; PRISMA ${ }^{[45]}$ and MOOSE ${ }^{[50]}$ guidelines for systematic reviews and meta-analysis of interventional studies and observational studies, respectively. Internet resources such as the Equator Network website (http://www.equator-network.org/) may be referred for enhancing the updates on quality and transparency of health research. Following approval from Institutional Ethical Committee and fulfilling ICMJE requirements, the clinical trials must be registered with the Clinical Trials Registry - India (CTRI; www.ctri.nic.in), which is a WHO International Clinical Trials Registry Platform. ${ }^{[19,51,52]}$ Further, reviewers should remain vigilant about research reports funded by pharmaceutical companies. There are few recognized hints about predatory journals which researchers should suspect and remain vigilant while submission of research paper. These predatory journals usually promises publishing in unusual time (i.e., days or weeks), charges heavy processing fee, does not have well-recognized affiliation, invites article personally by e-mail, name of journal is usually broad (e.g., "Journal of Sciences" or "Journal of Case Reports" or "International Journal...") to attract more submissions, often these are early issues but still claims high impact factor (whereas authentic journals usually receive impact factor after 2 years of publishing) ${ }^{[43]}$ Further, there should be regularly updated blacklist of these predatory journals so that young researchers before submission can check on national or government portals.

\section{Placebo}

Placebo response in depression studies ranged from $10 \%$ to $50 \%$ with an average of $30 \%$. This implies that even inactive treatment may show a significant response. Therefore, it raises doubt over results of nonplacebo studies. There are concerns in selecting inactive placebo in nonpharmacological modes of treatment.

\section{Recommendations}

Placebo-controlled studies need to be considered over uncontrolled studies. However, concern about placebo-induced worsening of symptoms had led to suggestions that any new psychoactive medication should be studied against an active comparator only. ${ }^{[3,54]}$ 


\section{CONCLUSION}

Continued research is essential to improve knowledge and practice of science. Ethics is heart of any research which balances rights of participant in keeping up with interest of research. Unethical medical research has grown over decades. This poses increased risk to vulnerable group including person with mental illness. No guidelines are currently available for this group of population. Psychiatry research has several specific issues which need to be taken care of and researchers need to be trained specifically for these issues. Although the current paper has highlighted various issues and recommendations, further this opens a topic for further consideration of other research issues in psychiatry as special subgroups (fetal, maternal, pediatric, geriatrics, poor, illiterate, homeless, linguistically distinct), subareas (genetic, addiction, internet based, telepsychiatry), procedures (brain stimulation techniques, pharmacological, psychotherapy, alternative therapy, and meditation) to frame a national guidelines for psychiatric research.

\section{REFERENCES}

1. MacKenzie CR. What would a good doctor do? Reflections on the ethics of medicine. HSS J 2009;5:196-9.

2. Armstrong JS, Sperry T. The ombudsman: Business school prestige-Research versus teaching. Interfaces 1994;24:13-43.

3. Demyttenaere K, Bruffaerts R, Posada-Villa J, Gasquet I, Kovess V, Lepine JP, et al. Prevalence, severity, and unmet need for treatment of mental disorders in the World Health Organization World Mental Health Surveys. JAMA 2004;291:2581-90.

4. Ministry of Health (MOH). National Mental Health Policy of India: New Pathways, New Hope. New Delhi: Ministry of Health, Government of India; 2014. Available from: http:// www.mohfw.nic.in/index1.php?lang=1\&level=2\&sublinki d=4723\&lid=2964. [Last cited on 2016 Feb 01].

5. Math SB, Srinivasaraju R. Indian Psychiatric epidemiological studies: Learning from the past. Indian J Psychiatry 2010;52 Suppl 1:S95-103.

6. World Health Organization. The Global Burden of Disease. Cambridge, Mass: Harvard University Press, 1997.

7. Sadock BJ, Sadock VA, Ruiz P, Kaplan HI. Kaplan \& Sadock's Comprehensive Textbook of Psychiatry. Philadelphia: Wolters Kluwer Health/Lippincott Williams \& Wilkins; 2009.

8. Weindling P. The origins of informed consent: The International Scientific Commission on Medical War Crimes, and the Nuremburg code. Bull Hist Med 2001;75:37-71.

9. Harkness JM. Nuremberg and the issue of wartime experiments on US prisoners. The Green Committee. JAMA 1996;276:1672-5. 


\section{Ethics in Psychiatric Research: Issues and Recommendations}

10. WMA Declaration of Helsinki: Ethical Principles for Medical Research Involving Human Subjects. Available from: http:// www.wma.net/en/30publications/10policies/b3/index. html. [Last cited on $2016 \mathrm{Feb} 26]$.

11. Katz RV, Kegeles SS, Kressin NR, Green BL, Wang MQ, James SA, et al. The Tuskegee Legacy Project: Willingness of minorities to participate in biomedical research. J Health Care Poor Underserved 2006;17:698-715.

12. Council for International Organization of Medical Sciences. International Ethical Guidelines for Biomedical Research Involving Human Subjects. Geneva: Council for International Organizations of Medical Sciences; 1993.

13. Strech D, Metz C, Knüppel H. Do editorial policies support ethical research? A thematic text analysis of author instructions in psychiatry journals. PLoS One 2014;9:e97492.

14. New Delhi: Indian Council of Medical Research. Ethical Guidelines for Biomedical Research on Human Subjects. 2006.

15. Agarwal AK,Gupta SC. Ethics in Psychiatry in, Vyas JN, Ahuja N (eds). Textbook of Postgraduate Psychiatry, 2nd ed. New Delhi : Jaypee Brothers Medical Publishers Ltd. 1999; p. 1019-25.

16. Chaturvedi SK. A review of Indian publications on ethical issues regarding capacity, informed consent, and placebo controlled trials. Internet Journal of Mental Health. 2008; 5.

17. Zhang D, Freemantle N, Cheng KK. Are randomized trials conducted in China or India biased? A comparative empirical analysis. J Clin Epidemiol 2011;64:90-5.

18. Balhara YP, Mishra A. A study exploring attributes and nature of the retracted literature on mental disorders. Indian J Med Ethics 2015;12:30-7.

19. Tharyan P, Premkumar TS, Mathew V, Barnabas JP, Manuelraj. Editorial policy and the reporting of randomized controlled trials: A survey of instructions for authors and assessment of trial reports in Indian medical journals (2004-2005). Natl Med J India 2008;21:62-8.

20. Chaturvedi SK, Somashekar BS. Reporting ethical aspects in published research articles in the Indian Journal of Psychiatry. Indian J Psychiatry 2009;51:34-7.

21. Dresser R. Mentally disabled research subjects. The enduring policy issues. JAMA 1996; 276:67-72.

22. Beauchamp TL, Childress JF. Principles of Biomedical Ethics. USA: Oxford University Press; 2001.

23. Math SB, Viswanath B, Maroky AS. Ethical issues in psychiatry in Southeast Asia: Research and practice. In: Trivedi JK, Tripathi A, editors. Mental Health in South Asia: Ethics, Resources, Programs and Legislation. New York/ London: Springer; 2014.

24. Shah R, Basu D. Coercion in psychiatric care: Global and Indian perspective. Indian J Psychiatry 2010; 52:203-6. 


\section{Ethics in Psychiatric Research: Issues and Recommendations}

25. Quitkin FM. Placebos, drug effects, and study design: A clinician's guide. Am J Psychiatry 1999;156:829-36.

26. López-Ibor JJ, López-Ibor MI, Helmchen H. Ethics of diagnosis and classification in psychiatry. In: Helmchen H, Sartorius N, editors. Ethics in Psychiatry. Springer: Netherlands; 2010. p. 199-207.

27. Stanley B, Stanley M. Psychiatric patients' comprehension of consent information. Psychopharmacol Bull 1987;23:375-8.

28. Henderson GE, Churchill LR, Davis AM, Easter MM, Grady C, Joffe S, et al. Clinical trials and medical care: Defining the therapeutic misconception. PLoS Med 2007;4:e324.

29. Appelbaum PS, Lidz CW. Twenty-five years of therapeutic misconception. Hastings Cent Rep 2008;38:5-6.

30. Henderson GE, Easter MM, Zimmer C, King NM, Davis AM, Rothschild BB, et al. Therapeutic misconception in early phase gene transfer trials. Soc Sci Med 2006; 62:239-53.

31. Dunn LB, Candilis PJ, Roberts LW. Emerging empirical evidence on the ethics of schizophrenia research. Schizophr Bull 2006;32:47-68.

32. Appelbaum PS, Grisso T. MacArthur Competence Assessment Tool for Clinical Research (MacCAT-CR). Professional Resource Press/Professional Resource Exchange; 2001. Available from: http://www.psycnet.apa. org/psycinfo/2001-18155-000. [Last cited on 2016 Mar 08].

33. Jeste DV, Palmer BW, Appelbaum PS, Golshan S, Glorioso D, Dunn LB, et al. A new brief instrument for assessing decisional capacity for clinical research. Arch Gen Psychiatry 2007;64:966-74.

34. Roberts LW, Roberts B. Psychiatric research ethics: An overview of evolving guidelines and current ethical dilemmas in the study of mental illness. Biol Psychiatry 1999;46:1025-38.

35. Candia PC, Barba AC. Mental capacity and consent to treatment in psychiatric patients: The state of the research. Curr Opin Psychiatry 2011;24:442-6.

36. Silva MC, Sorrell JM. Enhancing comprehension of information for informed consent: A review of empirical research. IRB 1988;10:1-5.

37. Palmer BW, Savla GN, Roesch SC, Jeste DV. Changes in capacity to consent over time in patients involved in psychiatric research. Br J Psychiatry 2013;202:454-8.

38. WMA Declaration of Helsinki: Ethical Principles for Medical Research Involving Human Subjects; 2013. Available from: http://www.wma.net/en/30publications/10policies/b3/ index.html. [Last cited on 2016 Feb 26].

39. Siriwardhana C, Adikari A, Jayaweera K, Sumathipala A. Ethical challenges in mental health research among internally displaced people: Ethical theory and research implementation. BMC Med Ethics 2013;14:13. 


\section{Ethics in Psychiatric Research: Issues and Recommendations}

40. Rothman DJ, McDonald WJ, Berkowitz CD, Chimonas SC, DeAngelis CD, Hale RW, et al. Professional medical associations and their relationships with industry: A proposal for controlling conflict of interest. JAMA 2009;301:1367-72.

41. Fang FC, Casadevall A. Retracted science and the retraction index. Infect Immun 2011; 79:3855-9.

42. Healy DT. Transparency and trust: Figure for ghost written articles was misquoted. BMJ 2004; 329:1345.

43. Bhad R, Hazari N. Predatory journals in psychiatry: A note of caution. Asian J Psychiatr 2015;16:67-8.

44. Schulz KF, Altman DG, Moher D; CONSORT Group. CONSORT 2010 Statement: Updated guidelines for reporting parallel group randomised trials. BMC Med 2010;8:18.

45. Moher D, Liberati A, Tetzlaff J, Altman DG; PRISMA Group. Preferred reporting items for systematic reviews and meta-analyses: The PRISMA statement. Ann Intern Med 2009;151:264-9, W64.

46. von Elm E, Altman DG, Egger M, Pocock SJ, Gøtzsche PC, Vandenbroucke JP; STROBE Initiative. The Strengthening the Reporting of Observational Studies in Epidemiology (STROBE) statement: Guidelines for reporting observational studies. Prev Med 2007;45:247-51.

47. Vandenbroucke JP, von Elm E, Altman DG, Gøtzsche PC, Mulrow CD, Pocock SJ, et al. Strengthening the Reporting of Observational Studies in Epidemiology (STROBE): Explanation and elaboration. Ann Intern Med 2007;147:W163-94.

48. Bossuyt PM, Reitsma JB, Bruns DE, Gatsonis CA, Glasziou PP, Irwig LM, et al. Towards complete and accurate reporting of studies of diagnostic accuracy: The STARD initiative. Clin Chem Lab Med 2003;41:68-73.

49. Tong A, Sainsbury P, Craig J. Consolidated criteria for reporting qualitative research (COREQ): A 32-item checklist for interviews and focus groups. Int J Qual Health Care 2007;19:349-57.

50. Stroup DF, Berlin JA, Morton SC, Olkin I, Williamson GD, Rennie D, et al. Meta-analysis of observational studies in epidemiology: A proposal for reporting. Meta-analysis Of Observational Studies in Epidemiology (MOOSE) Group. JAMA 2000; 283:2008-12.

51. Pandey A, Aggarwal A, Seth S, Maulik M, Bano R, Juneja A. Clinical Trials Registry India: Redefining the conduct of clinical trials. Indian J Cancer 2008;45:79-82.

52. Pandey A, Aggarwal AR, Maulik M, Seth SD. Clinical Trials Registry-India: Raising the veil. Natl Med J India 2010;23:187-8.

53. Walsh BT, Seidman SN, Sysko R, Gould M. Placebo response in studies of major depression: Variable, substantial, and growing. JAMA 2002;287:1840-7. 
Ethics in Psychiatric Research: Issues and Recommendations

54. Khan A, Detke M, Khan SR, Mallinckrodt C. Placebo response and antidepressant clinical trial outcome. J Nerv Ment Dis 2003;191:211-8.

How to cite this article: A Malla, N Bhat (2016), Ethics in Psychiatric Research: Issues and Recommendations, International Journal of Indian Psychology, Volume 3, Issue 4, No. 68, ISSN:2348-5396 (e), ISSN:2349-3429 (p), DIP:18.01.218/20160304, ISBN:978-1-365-39398-3 